\title{
Borassus flabellifer fruit flesh derived hierarchical porous partly graphitic carbon as a sustainable electrode for supercapacitor
}

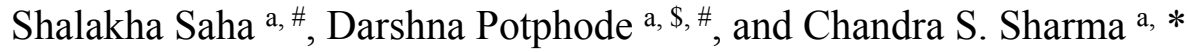 \\ ${ }^{a}$ Creative \& Advanced Research Based On Nanomaterials (CARBON) Laboratory, \\ Department of Chemical Engineering, Indian Institute of Technology, Hyderabad, \\ Kandi 502285, Telangana, India \\ $\$$ Presently at Department of Chemistry, University of Mumbai, Vidyanagari-Kalina, \\ Santacruz (East), Mumbai-400 098, India.
}

*Corresponding author e-mail address [cssharma@che.iith.ac.in]

\#S.S. and D.P. contributed equally to this paper.

\section{Supporting Information:}

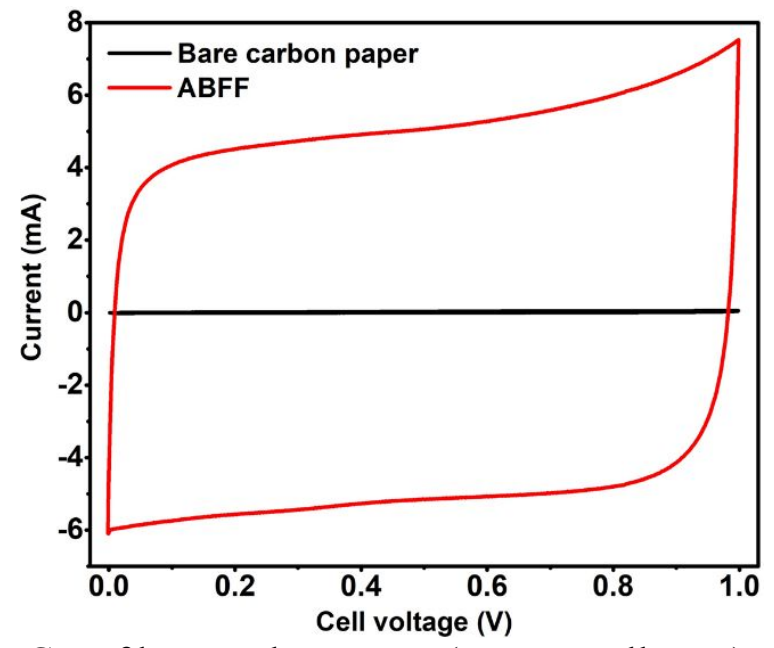

Figure S1. Comparative CV of bare carbon paper (current collector) and ABFF carbon-coated carbon paper in $1 \mathrm{M} \mathrm{H}_{2} \mathrm{SO}_{4}$ electrolyte at $25 \mathrm{mV} \mathrm{s}^{-1}$ indicating negligible charge storage contribution from the current collector. 

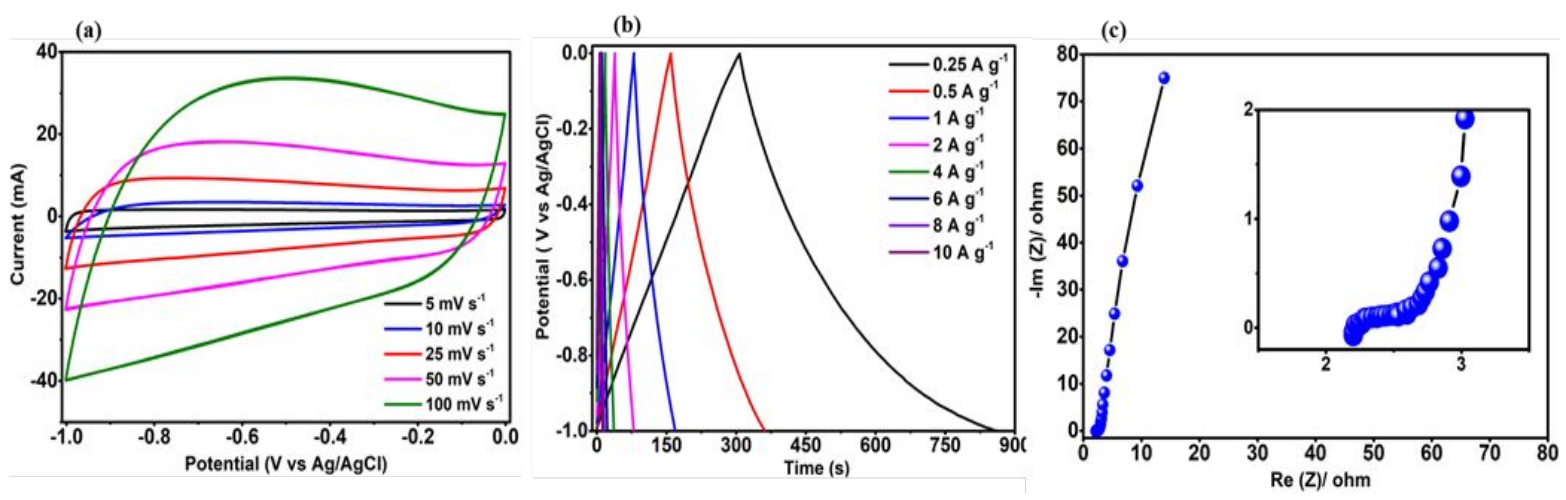

Figure S2. Electrochemical measurement of ABFF carbon in $1 \mathrm{M} \mathrm{H}_{2} \mathrm{SO}_{4}$ using a threeelectrode system.
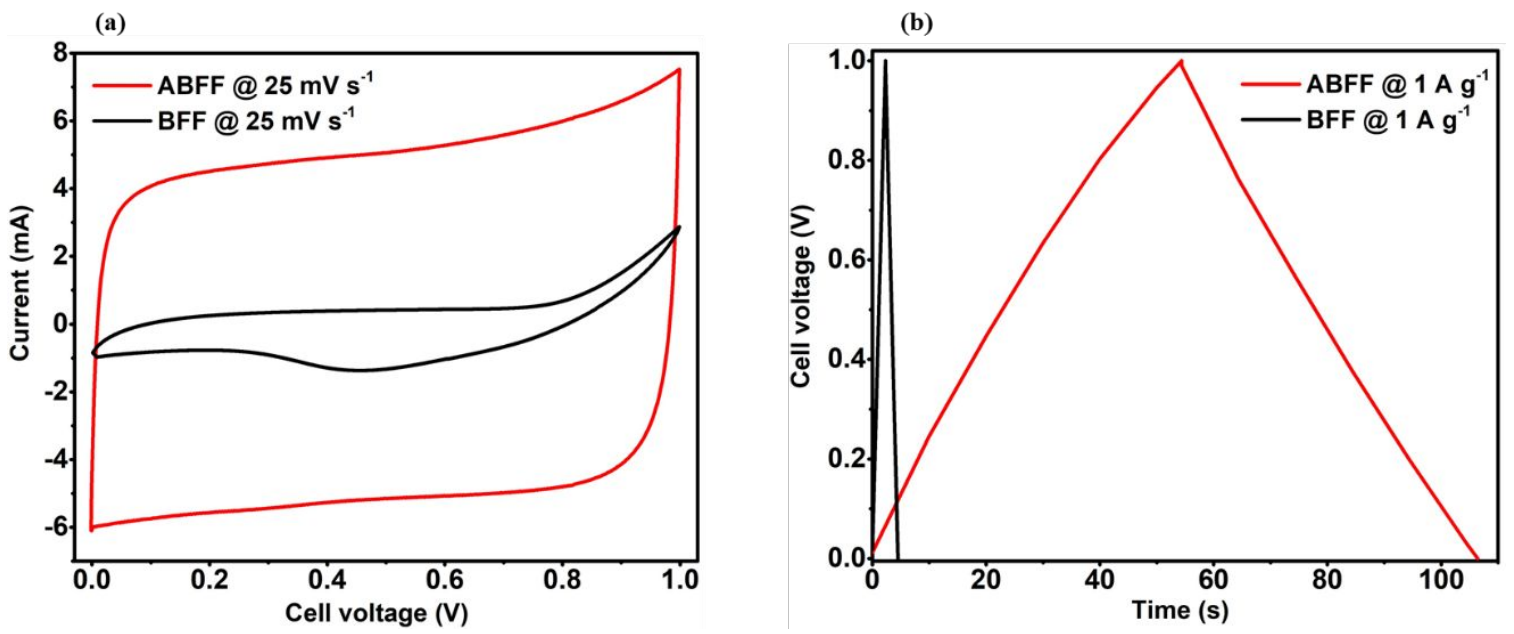

Figure S3. Comparative electrochemical performance of BFF (only carbonized) and ABFF carbon (BFF carbon after activation) symmetric device in $1 \mathrm{M} \mathrm{H}_{2} \mathrm{SO}_{4}$ : (a) $\mathrm{CV}$ curve at $25 \mathrm{mV}$ $\mathrm{s}^{-1}$ (b) GCD curve at $1 \mathrm{~A} \mathrm{~g}^{-1}$.
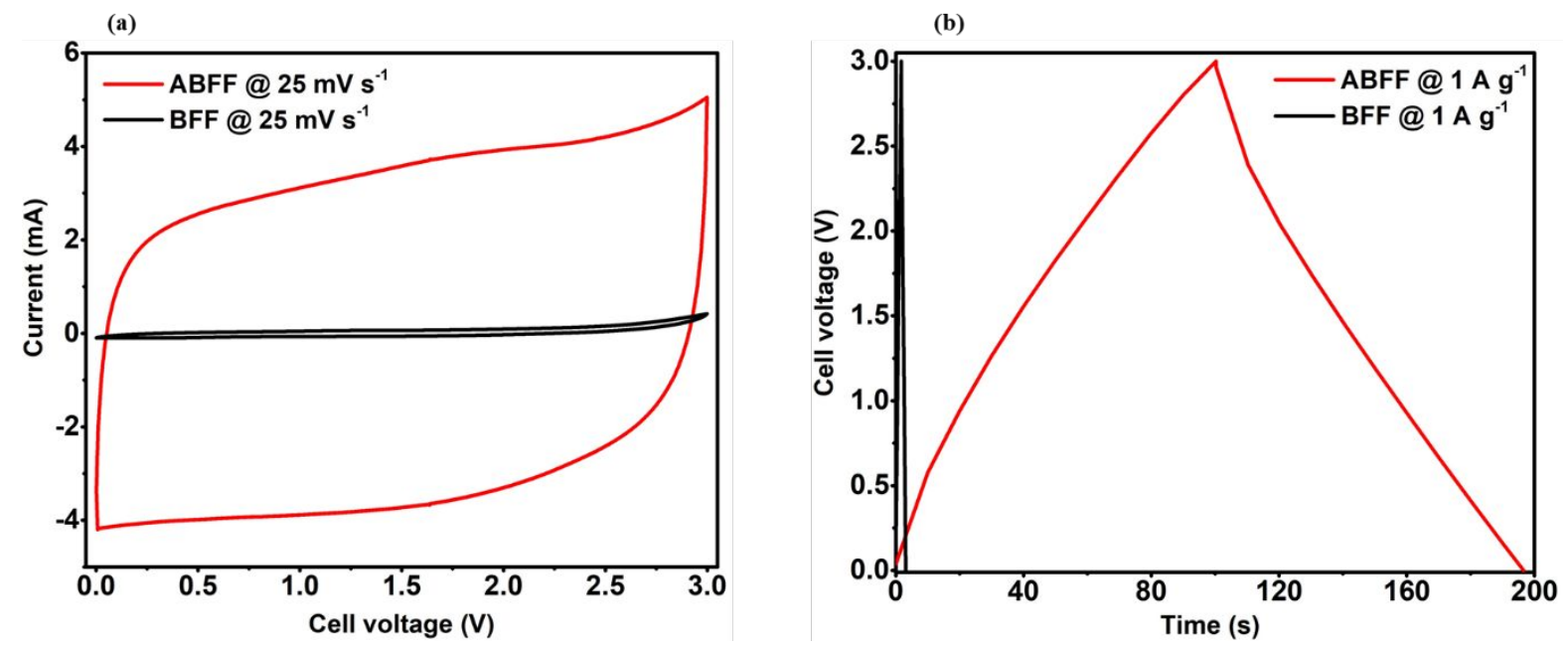

Figure S4. Comparative electrochemical performance of BFF (only carbonized) and ABFF carbon (BFF carbon after activation) symmetric device in neat ionic liquid (IL) EMIMBF 4 : (a) $\mathrm{CV}$ curve at $25 \mathrm{mV} \mathrm{s}^{-1}$ (b) GCD curve at $1 \mathrm{~A} \mathrm{~g}^{-1}$. 


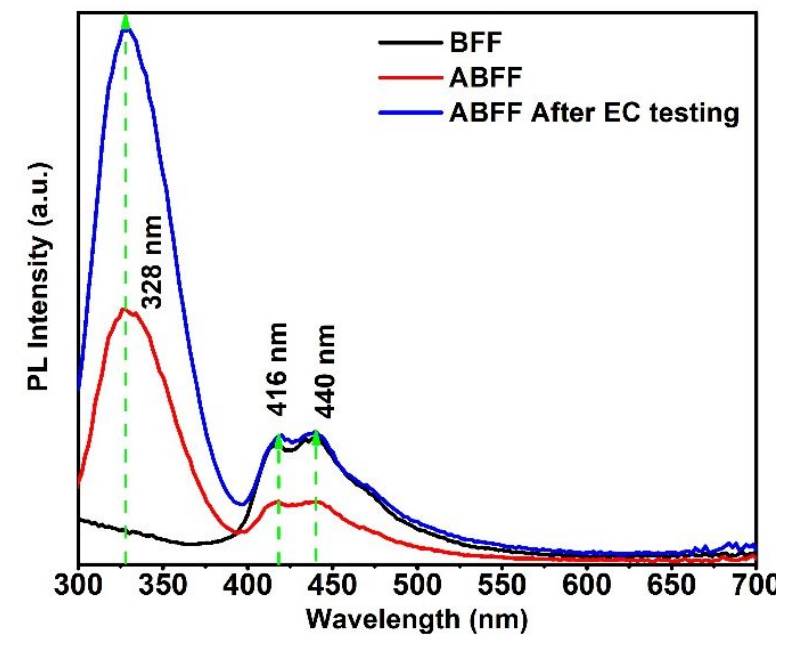

Figure S5. PL spectra of BFF carbon, ABFF carbon before and after electrochemical (EC) testing in $1 \mathrm{M} \mathrm{H}_{2} \mathrm{SO}_{4}$

(a)

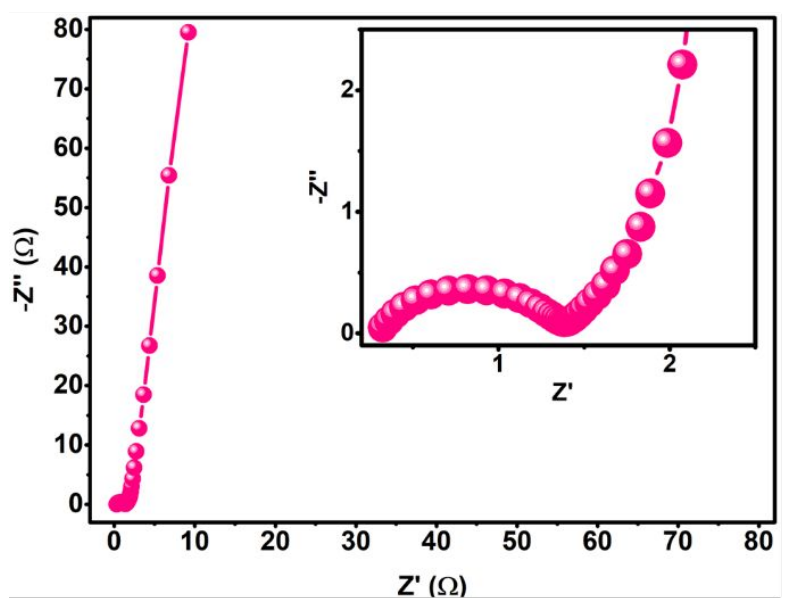

(b)

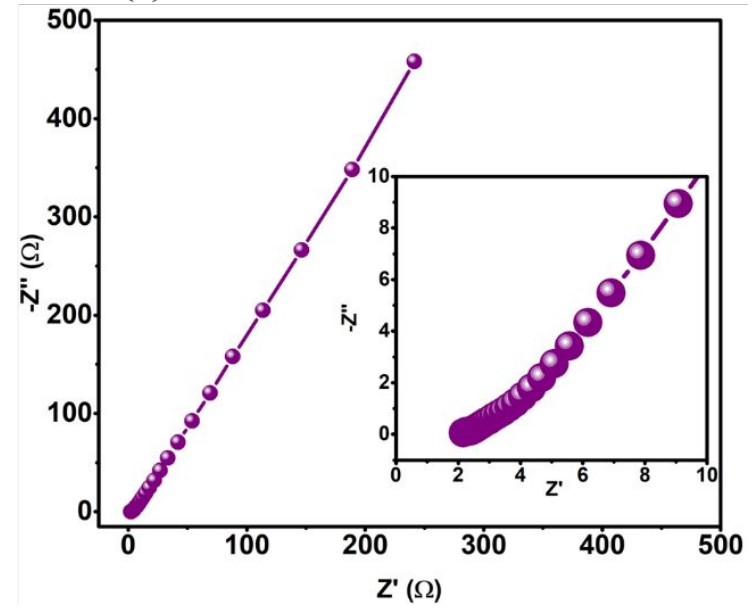

Figure S6. EIS curve of ABFF carbon symmetric device: (a) in $1 \mathrm{M} \mathrm{H}_{2} \mathrm{SO}_{4}$ (b) neat ionic liquid (IL) $\mathrm{EMIMBF}_{4}$ inset shows the magnified high-frequency region. 